\title{
Yttrium Y 90 Anti-CDH3 Monoclonal Antibody FF-21101
}

National Cancer Institute

\section{Source}

National Cancer Institute. Yttrium Y 90 Anti-CDH3 Monoclonal Antibody FF-21101. NCI

Thesaurus. Code C122405.

A radioimmunoconjug ate consisting of a chimeric monoclonal antibody targeting human cadherin-3 (CDH3) and labeled, via the macrocyclic chelator 1,4,7,10-

tetraazacyclododecane-1,4,7,10-tetraacetic acid (DOTA), with the beta-emitting radioisotope yttrium $Y 90$, with potential antineoplastic activities. Upon administration, the antibody moiety of yttrium Y 90 anti-CDH3 monoclonal antibody FF-21101 binds to CDH3 expressed on tumor cells, thereby specifically delivering cytotoxic beta radiation to $\mathrm{CDH} 3$-expressing tumor cells. $\mathrm{CDH} 3$, also known as P-cadherin, is a tumor-associated antigen (TAA) and member of the cadherin family; it is overexpressed in a variety of tumors and plays a role in cell adhesion, motility, invasion, and proliferation. 